\title{
DERIVATIONS AND AUTOMORPHISMS OF NONASSOCIATIVE MATRIX ALGEBRAS ${ }^{1}$
}

\author{
BY
}

\author{
G. M. BENKART AND J. M. OSBORN
}

\begin{abstract}
This paper studies the derivation algebra and the automorphism group of $M_{n}(A), n \times n$ matrices over an arbitrary nonassociative algebra $A$ with multiplicative identity 1 . The investigation also includes results on derivations and automorphisms of the algebras obtained from $M_{n}(A)$ using the Lie product $[x y]=$ $x y-y x$, and the Jordan product $x \circ y=\frac{1}{2}(x y+y x)$.
\end{abstract}

1. Introduction. Results concerning automorphisms and derivations of $n \times n$ matrices over a finite dimensional central associative division algebra have been known for some time. The Noether-Skolem theorem combined with a theorem due to Jacobson gives that automorphisms as well as derivations of these algebras are inner. This present paper generalizes these theorems by considering derivations and automorphisms of $M_{n}(A), n \times n$ matrices over an arbitrary nonassociative algebra $A$ with 1 . The investigation also determines the derivations and automorphisms of the algebras obtained from $M_{n}(A)$ using the Lie and Jordan products. With one exception the derivation algebras are shown to all follow the same pattern. They consist of inner derivations by matrices with entries in the nucleus $N$ of $A$, and of derivations gotten by applying derivations of $A$ to each matrix entry. Every derivation is the sum of these two kinds of derivations. More variability arises in the automorphism groups. The common feature is a subgroup composed of conjugations by invertible matrices in $M_{n}(N)$, and of automorphisms obtained by applying automorphisms of $A$ entry by entry. Each element of this subgroup is a product of these two kinds of maps.

Our motivation to investigate such derivation algebras and automorphism groups was due in part to conversations with physicists concerned with building algebraic models in particle theories. Of particular interest to them were the cases when $A$ was taken to be an octonion algebra or a certain 7-dimensional noncommutative Jordan algebra [1]. The results we present here have already been used in a negative sense to exclude some algebras from their considerations.

The main results concerning the group of antiautomorphisms and automorphisms of $M_{n}(A)$ are contained in Corollary 3.14. Theorem 4.8 and Corollaries 4.9, 4.10 describe the derivation algebras of $M_{n}(A), L_{n}(A), L_{n}^{\prime}(A)$, and $K_{n}(A)$ where

Received by the editors October 20, 1978.

AMS (MOS) subject classifications (1970). Primary 16A72, 16A68, 17B40, 17C30, 17E05.

Key words and phrases. Derivations, automorphisms, antiautomorphisms, nonassociative algebras, nonassociative matrix algebras.

'Partially supported by N.S.F. grant MCS 77-01724. 
$L_{n}(A)$ is $M_{n}(A)$ under the product [ ], $L_{n}^{\prime}(A)=\left[L_{n}(A), L_{n}(A)\right]$ and $K_{n}(A)$ is $L_{n}^{\prime}(A)$ with its center factored out. We present results for the automorphism groups of $L_{n}(A), L_{n}^{\prime}(A)$, and $K_{n}(A)$ in Theorems 4.12 and 4.13, and Corollary 4.14. Finally, if $J_{n}(A)$ denotes $M_{n}(A)$ viewed under the Jordan product, then its derivation algebra is obtained in Theorem 5.5, and its automorphism group is described in Theorem 5.6.

2. Basic concepts. Let $A$ be an arbitrary nonassociative algebra with identity 1 over the field $\Phi$, and let $N$ denote the nucleus of $A$. That is

$$
N=\{a \in A \mid(a, b, c)=(b, a, c)=(b, c, a)=0 \text { for all } b, c \in A\}
$$

where $(a, b, c)=(a b) c-a(b c)$. The algebra $N$ is associative, and it is not difficult to show that if $a \in N$, then $\operatorname{ad}_{a}: A \rightarrow A$ given by $\operatorname{ad}_{a}(b)=[a b]=a b-b a$ is a derivation of $A$.

In the same manner we speak of the nucleus of $M_{n}(A)$. An easy calculation shows that it is $M_{n}(N)$, and as above for $x \in M_{n}(N), \operatorname{ad}_{x}$ is a derivation of $M_{n}(A)$. If Der $M_{n}(A)$ denotes the derivation algebra of $M_{n}(A)$, then $\operatorname{ad}_{M_{n}(N)}=\left\{\operatorname{ad}_{x} \mid x \in\right.$ $\left.M_{n}(N)\right\}$ is a subalgebra of $\operatorname{Der} M_{n}(A)$. Let $e_{i j}$ denote the matrix with 1 in the $(i, j)$ position and 0 elsewhere. Then these matrix units lie in $M_{n}(N)$, and the derivations $\operatorname{ad}_{e_{i j}}$ will be particularly useful in what follows.

In addition to the inner derivations $\operatorname{ad}_{M_{n}(N)}$ one can also obtain a derivation of $M_{n}(A)$ by beginning with a derivation of $A$ and applying it to each matrix entry. The resulting derivations form a subalgebra of $\operatorname{Der} M_{n}(A)$, which we denote by $(\operatorname{Der} A)^{\#}$. The notation is suggestive of the fact that $(\operatorname{Der} A)^{\#}$ is isomorphic to Der $A$. Similarly one can obtain an automorphism of $M_{n}(A)$ by applying an automorphism of $A$ to each entry. The resulting set of automorphisms is a subgroup, called (Aut $A)^{\#}$, of the full automorphism group Aut $M_{n}(A)$. If $u \in$ $M_{n}(N)$ is invertible then conjugation by $u$, denoted by $\chi_{u}\left(\chi_{u}(x)=u^{-1} x u\right)$, belongs to Aut $M_{n}(A)$. We write $\operatorname{GL}(n, N)$ for the subgroup composed of the mappings $\chi_{u}$.

One final note, let us observe that if $\varphi \in \operatorname{Aut} M_{n}(A)$ or $\varphi \in \operatorname{Der} M_{n}(A)$, then $\varphi$ maps $M_{n}(N)$ into $M_{n}(N)$. This is true since $M_{n}(N)$ is characterized as the nucleus of $M_{n}(A)$, and derivations and automorphisms preserve the nucleus.

3. Generalized automorphisms and antiautomorphisms of $M_{n}(A)$. Given any algebra $A$ with nucleus $N$ let $Z(A)=\{a \in N \mid[a b]=0$ for all $b \in A\}$ denote the center of $A$. We consider linear transformations $\varphi$ of $A$ onto $A$ with $\varphi(1)=1$ and with the following property:

There exist idempotents $f, g \in Z(A)$ such that $f+g=1, f g=0$, and if $f^{\prime}=$ $\varphi(f), g^{\prime}=\varphi(g)$, then $f^{\prime}, g^{\prime} \in Z(A)$ and $\varphi: f A \rightarrow f^{\prime} A$ is an algebra isomorphism and $\varphi: g A \rightarrow g^{\prime} A$ is an anti-isomorphism.

LEMMA 3.1. The set of all such mappings, GAut $A$, is a group.

Proof. Given $\varphi \in$ GAut $A$, then the condition holds for $\varphi^{-1}$ and the idempotents $f^{\prime}, g^{\prime}$. Suppose now $\varphi_{1}$ and $\varphi_{2}$ belong to GAut $A$ and the corresponding idempotents are $f_{1}, g_{1}$ and $f_{2}, g_{2}$. Then one can verify the above property is satisfied 
for $\varphi_{2} \varphi_{1}$ using the idempotents $f=f_{2} f_{1}^{\prime}+g_{2} g_{1}^{\prime}$ and $g=f_{2} g_{1}^{\prime}+g_{2} f_{1}^{\prime}$ where $f_{1}^{\prime}=$ $\varphi_{1}\left(f_{1}\right)$ and $g_{1}^{\prime}=\varphi_{1}\left(g_{1}\right)$.

The group GAut $\boldsymbol{A}$ contains two distinguished subgroups, Aut $\boldsymbol{A}$ and AAut $\boldsymbol{A}$, where AAut $A$ is the group consisting of all automorphisms and antiautomorphisms of $A$. Corresponding to each $\varphi \in$ GAut $A$ is the decomposition of $A$ into the direct sum of the ideals $f A$ and $g A$. So if $A$ has no proper direct summands (for example if $A$ is simple), then GAut $A=$ AAut $A$.

A straightforward calculation shows that each $\varphi \in$ GAut $A$ maps the nucleus of $A$ onto itself.

Following the procedure above we define GAut $M_{n}(A)$. The idempotents corresponding to elements in GAut $M_{n}(A)$ lie in $Z\left(M_{n}(A)\right)$ which is $Z(A) I$, where $I$ is the identity matrix. For each $\varphi \in$ GAut $M_{n}(A), \varphi\left(M_{n}(N)\right)=M_{n}(N)$ since $\varphi$ preserves the nucleus, and $\varphi$ restricted to $M_{n}(N)$ lies in GAut $M_{n}(N)$. In this section we investigate the structure of $\operatorname{GAut} M_{n}(A)$ under the assumption that $N$ is Artinian. As the preceding remarks indicate the place to begin is with GAut $M_{n}(N)$, but first some preliminary results brought to our attention by $\mathrm{L}$. Levy [7, Lemma 3.2, p. 282].

LEMMA 3.2. Let $R$ be an associative ring with 1 and $M$ be a right $R$-module such that

$$
M=M_{1} \oplus \cdots \oplus M_{n}=M_{1}^{\prime} \oplus \cdots \oplus M_{n}^{\prime}
$$

are two direct sum decompositions of $M$ into submodules such that $M_{i}$ is isomorphic to $M_{i}^{\prime}$ for $1 \leqslant i \leqslant n$. Then there is an invertible $\sigma$ in the ring of $R$-endomorphisms of $M$ such that $\sigma^{-1} \pi_{i}^{\prime} \sigma=\pi_{i}$ for each $1 \leqslant i \leqslant n$, where $\pi_{i}\left(\pi_{i}^{\prime}\right)$ denotes the projection of $M$ onto $M_{i}\left(M_{i}^{\prime}\right)$.

Proof. For each $i$ extend the isomorphism between $M_{i}$ and $M_{i}^{\prime}$ to an $R$-endomorphism of $M$ by defining it to be 0 on the other summands. Take $\sigma$ to be the sum of the resulting $n$ endomorphisms.

LemMa 3.3. Let $R$ be an associative ring with 1 and let $\left\{e_{i}\right\}$ and $\left\{f_{i}\right\}$ for $i=1, \ldots, n$ be two sets of orthogonal idempotents summing to 1 . Assume for each $i$, $e_{i} R$ is isomorphic to $f_{i} R$ as $R$-modules. Then there is a unit $u \in R$ with $u^{-1} f_{i} u=e_{i}$ for $i=1, \ldots, n$.

Proof. Apply the preceding lemma to

$$
R=e_{1} R \oplus \cdots \oplus e_{n} R=f_{1} R \oplus \cdots \oplus f_{n} R .
$$

The projection $\pi_{i}\left(\pi_{i}^{\prime}\right)$ is just left multiplication by $e_{i}\left(f_{i}\right)$. The $R$-isomorphism $\sigma$ is left multiplication by $u=\sigma(1)$, and $\sigma^{-1}$ is left multiplication by $u^{-1}$. Then

$$
e_{i}=\pi_{i}(1)=\sigma^{-1} \pi_{i}^{\prime} \sigma(1)=\sigma^{-1} \pi_{i}^{\prime}(u)=\sigma^{-1}\left(f_{i} u\right)=u^{-1} f_{i} u .
$$

Given $\varphi \in$ GAut $M_{n}(A)$ direct verification shows that $\varphi$ is an automorphism of $J_{n}(A)$, where $J_{n}(A)$ is $M_{n}(A)$ under the Jordan product $x \circ y=\frac{1}{2}(x y+y x)$ provided char $\Phi \neq 2$. It is convenient for what follows in $\$ 5$ to work with the larger group Aut $J_{n}(A)$. Though we disallow char $\Phi=2$ in this process, this restriction is unnecessary in dealing with GAut $M_{n}(A)$. 
Jacobson [4, Theorem 7.4, p. 26] has shown that as a consequence of results in either [4] or [6] the following theorem holds:

Let $A$ and $B$ be arbitrary algebras with identities, and let $B_{J}$ be the algebra obtained from $B$ under the Jordan product. Suppose $\psi: J_{n}(A) \rightarrow B_{J}$ is a Jordan homomorphism such that the images of the matrix units $e_{i j}$ lie in the nucleus of the algebra $C$ generated by $\psi\left(J_{n}(A)\right)$. Then $C=C_{1} \oplus C_{2}$ where the $C_{i}$ are ideals of $C$, and if $\pi_{i}$ denotes the projection of $C$ onto $C_{i}$, then $\pi_{i} \psi$ is a homomorphism, and $\pi_{2} \psi$ an antihomomorphism.

We could use this theorem to reduce our considerations to automorphisms and antiautomorphisms. However, we do not follow this line of attack because one virtue of working with GAut $M_{n}(A)$ is that it allows us to study automorphisms and antiautomorphisms simultaneously, and because we can obtain in the process results concerning automorphisms of $J_{n}(A)$ with the property that $\psi\left(e_{i i}\right)=e_{i i}$ for all $i$, without assuming from the outset that all the $\psi\left(e_{i j}\right)$ lie in the nucleus.

Lemma 3.4. Assume char $\Phi \neq 2$, and let $\varphi \in$ Aut $J_{n}(N)$, where $N$ is an associative Artinian ring with 1 . Then there is an invertible $u \in M_{n}(N)$ such that

$$
u^{-1} \varphi\left(e_{i i}\right) u=e_{i i}
$$

Proof. The elements $\varphi\left(e_{i i}\right)$ for $i=1, \ldots, n$ are orthogonal idempotents summing to 1 . Thus,

$$
\begin{aligned}
M_{n}(N) & =\varphi\left(e_{11}\right) M_{n}(N) \oplus \cdots \oplus \varphi\left(e_{n n}\right) M_{n}(N) \\
& =e_{11} M_{n}(N) \oplus \cdots \oplus e_{n n} M_{n}(N) .
\end{aligned}
$$

Jacobson and Rickart [6, Lemma 3, p. 487] have shown that there are matrix units $\left\{g_{i j}\right\}$ and $\left\{h_{i j}\right\}$ such that $\varphi\left(e_{i j}\right)=g_{i j}+h_{i j}$ and $g_{i j} h_{k l}=0=h_{k l} g_{i j}$ for all $i, j, k, l$. Therefore, $\varphi\left(e_{i i}\right) M_{n}(N)$ is isomorphic as an $M_{n}(N)$-module to $\varphi\left(e_{j j}\right) M_{n}(N)$ via left multiplication by $g_{j i}+h_{j i}$. Thus, $M_{n}(N)$ is isomorphic to the direct sum of $n$-copies of $\varphi\left(e_{i i}\right) M_{n}(N)$, and we write this $M_{n}(N) \approx \varphi\left(e_{i i}\right) M_{n}(N)^{(n)}$. Similarly $e_{i i} M_{n}(N)$ is isomorphic as a right $M_{n}(N)$-module to $e_{j j} M_{n}(N)$ using left multiplication by $e_{j i}$. Hence, $e_{i i} M_{n}(N)^{(n)} \approx M_{n}(N) \approx \varphi\left(e_{i i}\right) M_{n}(N)^{(n)}$. Breaking $e_{i i} M_{n}(N)$ into a finite number of indecomposable $M_{n}(N)$-modules, and collecting isomorphic indecomposables, one can use the Krull-Schmidt theorem to argue that $e_{i i} M_{n}(N)$ is isomorphic to $\varphi\left(e_{i i}\right) M_{n}(N)$. Thus by the preceding lemma, there is an invertible $u \in M_{n}(N)$ so that $u^{-1} \varphi\left(e_{i i}\right) u=e_{i i}$ as desired.

Corollary 3.5. Let $A$ be an algebra with 1 such that the nucleus $N$ of $A$ is Artinian. Then for each $\varphi \in$ GAut $M_{n}(A)$, there is an invertible $u \in M_{n}(N)$ so that $\psi=\chi_{u} \varphi \in$ GAut $M_{n}(A)$ has the property that $\psi\left(e_{i i}\right)=e_{i i}$ for $i=1, \ldots, n$.

Automorphisms of $J_{n}(A)$ with the property that $\psi\left(e_{i i}\right)=e_{i i}$ will be the topic of the next lemma, but one additional piece of notation is necessary.

Suppose $\theta \in$ GAut $A$ with idempotents $f, g$. Define $\theta^{\#}: M_{n}(A) \rightarrow M_{n}(A)$ by letting $\theta^{\#}$ on $f I \cdot M_{n}(A)$ be $\theta$ applied to each entry, and letting $\theta^{\#}$ on $g I \cdot M_{n}(A)$ be $\theta$ applied to each entry followed by taking the transpose of the resulting matrix. 
One can show $\theta^{\#} \in$ GAut $M_{n}(A)$. Let (GAut $\left.A\right)^{\#}$ denote the group of these mappings, and observe it is isomorphic to the group GAut $A$.

Theorem 3.6. Assume char $\Phi \neq 2$, and suppose $\psi \in$ Aut $J_{n}(A)$ with $\psi\left(e_{i i}\right)=e_{i i}$ for all $i=1, \ldots, n$. Then there is an invertible $v \in M_{n}(N)$ such that $\chi_{v} \psi \in$ (GAut $A)^{\#}$.

Proof. There are only five types of nonzero products in $J_{n}(A)$, and for $i, j, k$ distinct they are

$$
\begin{aligned}
a e_{i i} \circ b e_{i i} & =(a \circ b) e_{i i}, \\
a e_{i i} \circ b e_{i j} & =\frac{1}{2} a b e_{i j}, \\
a e_{i j} \circ b e_{j j} & =\frac{1}{2} a b e_{i j}, \\
a e_{i j} \circ b e_{j k} & =\frac{1}{2} a b e_{i k}, \\
a e_{i j} \circ b e_{j i} & =\frac{1}{2}\left(a b e_{i i}+b a e_{j j}\right) .
\end{aligned}
$$

We apply $\psi$ to these relations, and make different specializations of $a$ and $b$.

Relation (3.1) with $b=1$ says $\psi\left(a e_{i i}\right)=\psi\left(a e_{i i}\right) \circ e_{i i}$. But the first three equations above demonstrate the fact that the only elements of $J_{n}(A)$ which lie in the 1 -eigenspace relative to right multiplication by $e_{i i}$ are of the form $c e_{i i}$. Therefore

$$
\psi\left(a e_{i i}\right)=\psi_{i}(a) e_{i i} .
$$

Again by using (3.1) we see $\psi_{i}$ is an automorphism of $A$ under the product $a \circ b=\frac{1}{2}(a b+b a)$.

From (3.2) and (3.3) it follows that $\psi\left(a e_{i j}\right)$ must lie in $A e_{i j}+A e_{j i}$, which is the intersection of the $\frac{1}{2}$-eigenspace relative to multiplication by $e_{i i}$ with the $\frac{1}{2}$-eigenspace relative to multiplication by $e_{i j}$. As a special case of this fact:

$$
\psi\left(e_{i j}\right)=\alpha_{i j} e_{i j}+\beta_{j i} e_{j i} \text {. }
$$

It follows directly from $e_{i j} \circ e_{i j}=0$ that

$$
\alpha_{i j} \beta_{j i}=0=\beta_{j i} \alpha_{i j} \text {. }
$$

Now using (3.4) and (3.5) with $a=b=1$, we deduce further results concerning the $\alpha$ 's and $\beta$ 's, namely

$$
\begin{gathered}
\alpha_{i j} \alpha_{j k}=\alpha_{i k}, \quad \beta_{k j} \beta_{j i}=\beta_{k i}, \\
\alpha_{i j} \alpha_{j i}+\beta_{i j} \beta_{j i}=1 .
\end{gathered}
$$

If $\psi$ belongs to GAut $M_{n}(A)$, then it is immediate that $\alpha_{i j}, \beta_{j i} \in N$. We do not make this assumption in order to use Theorem 3.6 in $\$ 5$. Instead the fact that $\alpha_{i j}, \beta_{j i} \in N$ will be a consequence of the next few steps.

LEMMA 3.7. $\alpha_{i j}, \beta_{j i}$ lie in the middle nucleus of $A$ for all $i \neq j$.

Proof. If $\psi$ is applied to the relation $0=\left(a e_{i i} \circ e_{i j}\right) \circ b e_{j j}-a e_{i i} \circ\left(e_{i j} \circ b e_{j j}\right)$, then the $(i, j)$ component of the resulting equation shows that the associator $\left(\psi_{i}(a), \alpha_{i j}, \psi_{j}(b)\right)=0$, and the $(j, i)$ component shows that $\left(\psi_{j}(b), \beta_{j i}, \psi_{i}(a)\right)=0$. Since $\psi_{i}, \psi_{j}$ are onto, the proof is complete. 
Let us define $f_{i}=\alpha_{i j} \alpha_{j i}$ and $g_{i}=\beta_{i j} \beta_{j i}$. Note as a result of Lemma 3.7 and (3.9), if $n \geqslant 3$ then

$$
f_{i}=\alpha_{i j} \alpha_{j i}=\alpha_{i k} \alpha_{k j} \alpha_{j i}=\alpha_{i k} \alpha_{k i}
$$

This shows $f_{i}$ does not depend on the $j$ used to define it as long as $j \neq i$. Similarly $g_{i}$ is also independent of $j$.

LEMMA 3.8. For each $i, f_{i}$ and $g_{i}$ are orthogonal idempotents summing to 1 , and they commute with the elements of $A$. Moreover $f_{i}=f_{j}, g_{i}=g_{j}$ for all $i, j$.

Proof. Equation (3.9) says $f_{i}+g_{i}=1$. Now

$$
f_{i}^{2}=\alpha_{i j} \alpha_{j i} \alpha_{i j} \alpha_{j i}=\alpha_{i j}\left(1-\beta_{j i} \beta_{i j}\right) \alpha_{j i}=\alpha_{i j} \alpha_{j i}=f_{i},
$$

so that $f_{i}$ and $g_{i}=1-f_{i}$ are idempotents. Multiplying $f_{i}+g_{i}=1$ on the right by $f_{i}$, then on the left, gives $f_{i}^{2}+g_{i} f_{i}=f_{i}$ and $f_{i}^{2}+f_{i} g_{i}=f_{i}$. Thus $g_{i} f_{i}=0=f_{i} g_{i}$ and they are orthogonal.

To obtain the rest of the conclusions we return to (3.2), set $b=1$, and act on the equation with $\psi$. The result is a formula for $\psi\left(a e_{i j}\right)$

$$
\psi\left(a e_{i j}\right)=\psi_{i}(a) \alpha_{i j} e_{i j}+\beta_{j i} \psi_{i}(a) e_{j i}
$$

Using this formula let us calculate the $(i, i)$-coefficient of both sides of

$$
2 \psi\left(a e_{i i}+a e_{j j}\right) \circ \psi\left(e_{j i}\right)
$$

to establish that $\psi_{i}(a)=\psi_{i}(a) \alpha_{i j} \alpha_{j i}+\beta_{i j} \beta_{j i} \psi_{i}(a)=\psi_{i}(\alpha) f_{i}+g_{i} \psi_{i}(a)$. Since $\psi_{i}$ is onto, we can replace $\psi_{i}(a)$ with $a$ to obtain

$$
a=a f_{i}+g_{i} a \text { for all } a \in A \text {. }
$$

Multiplying $1=f_{i}+g_{i}$ on the right by $a$ shows that $a=f_{i} a+g_{i} a$. Comparing this result with (3.12) gives $a f_{i}=f_{i} a$ for all $a \in A$, and hence $g_{i} a=a g_{i}$ also.

Finally $f_{i}=f_{i}^{2}=\alpha_{i j} \alpha_{j i} \alpha_{i j} \alpha_{j i}=\alpha_{i j} f_{j} \alpha_{j i}=\alpha_{i j} \alpha_{j i} f_{j}=f_{i} f_{j}$. Since this is true for each $i$ and $j, f_{j}=f_{j} f_{i}$. But $f_{i}$ commutes with every element of $A$, so $f_{j}=f_{j} f_{i}=f_{i} f_{j}=f_{i}$. Thus $g_{i}=1-f_{i}=1-f_{j}=g_{j}$ and the proof is finished.

We shall just write $f^{\prime}$ for $f_{i}$ and $g^{\prime}$ for $g_{i}$ hereafter.

Lemma 3.9. The elements $\alpha_{i j}$ and $\beta_{j i}$ lie in $N$ for every pair $i, j$ with $i \neq j$.

Proof. In analogy with equation (3.11), if $\psi$ is applied to (3.3) with $a=1$, we obtain

$$
\psi\left(b e_{i j}\right)=\alpha_{i j} \psi_{j}(b) e_{i j}+\psi_{j}(b) \beta_{j i} e_{j i}
$$

We freely use these two equations in the proof of this lemma. First, let us apply $\psi$ to $\left(a e_{i j} \circ b e_{i i}\right) \circ e_{i j}-a e_{i j} \circ\left(b e_{i i} \circ e_{i j}\right)=0$. The $(j, j)$ coefficient is

$$
\begin{aligned}
\left(\left(\beta_{j i} \psi_{i}(a)\right)\right. & \left.\psi_{i}(b)\right) \alpha_{i j}+\beta_{j i}\left(\psi_{i}(b)\left(\psi_{i}(a) \alpha_{i j}\right)\right) \\
& -\left(\beta_{j i} \psi_{i}(a)\right)\left(\psi_{i}(b) \alpha_{i j}\right)-\left(\beta_{j i} \psi_{i}(b)\right)\left(\psi_{i}(a) \alpha_{i j}\right)=0 .
\end{aligned}
$$

Since $\psi_{i}$ is onto, we can replace $\psi_{i}(b)$ with any element $c$ in $A$, and $\psi_{i}(a)$ with an arbitrary element of $A$, so let us use $\left(\beta_{i j} d\right) g^{\prime}$. As a consequence of (3.8) and Lemma $3.8, g^{\prime} \alpha_{i j}=\beta_{i j} \beta_{j i} \alpha_{i j}=0$, and thus, $\left(\left(\beta_{i j} d\right) g^{\prime}\right) \alpha_{i j}=0$. Now 


$$
\begin{aligned}
\beta_{j i}\left(\left(\beta_{i j} d\right) g^{\prime}\right) & =\beta_{j i}\left(g^{\prime}\left(\beta_{i j} d\right)\right)=\left(\beta_{j i} g^{\prime}\right)\left(\beta_{i j} d\right) \\
& =\left(g^{\prime} \beta_{j i}\right)\left(\beta_{i j} d\right)=\left(g^{\prime} \beta_{j i} \beta_{i j}\right) d=g^{\prime} d,
\end{aligned}
$$

so that from this judicious choice of $\psi_{i}(a)$, we deduce

$$
\left(g^{\prime} d, c, \alpha_{i j}\right)=0 .
$$

If in the above equation the substitution of $f^{\prime}\left(d \alpha_{j i}\right)$ for $\psi_{i}(a)$ is made, the result is

$$
\left(\beta_{j i}, c, f^{\prime} d\right)=0 .
$$

To obtain the other half of the proof that $\alpha_{i j}$ is in the right nucleus and $\beta_{j i}$ in the left, we apply $\psi$ to the relation $\left(a e_{j i} \circ b e_{i i}\right) \circ e_{i j}-a e_{j i} \circ\left(b e_{i i} \circ e_{i j}\right)=\frac{1}{4}[a b] e_{i i}$. The $(j, j)$ coefficient of the left side must be 0 , and in the resulting equation for the $(j, j)$ coefficient we perform the replacements of $\psi_{i}(b)$ with $c$, and $\psi_{i}(a)$ with $\left(\alpha_{i j} d\right) f^{\prime}$ then with $g^{\prime}\left(d \beta_{j i}\right)$. The effect is

$$
\begin{aligned}
& \left(f^{\prime} d, c, \alpha_{i j}\right)=0 . \\
& \left(\beta_{j i}, c, g^{\prime} d\right)=0 .
\end{aligned}
$$

From relations (3.14)-(3.17) we conclude that $\alpha_{i j}$ is in the right nucleus and $\beta_{j i}$ in the left. The appropriate equations to use to achieve $\alpha_{i j}$ in the left nucleus and $\beta_{j i}$ in the right are

$$
\begin{aligned}
& \left(e_{i j} \circ a e_{j j}\right) \circ b e_{i j}-e_{i j} \circ\left(a e_{i j} \circ b e_{i j}\right)=0, \\
& \left(e_{i j} \circ a e_{j j}\right) \circ b e_{j i}-e_{i j} \circ\left(a e_{j j} \circ b e_{j i}\right)=\frac{1}{4}[b a] e_{i j} .
\end{aligned}
$$

The substitutions needed in the first equation are $g^{\prime}\left(d \beta_{i j}\right)$ and $\left(\alpha_{j i} d\right) f^{\prime}$ for $\psi_{j}(b)$, and in the second $f^{\prime}\left(d \alpha_{i j}\right)$ and $\left(\beta_{j i} d\right) g^{\prime}$ for $\psi_{j}(b)$.

Corollary 3.10. $f^{\prime}, g^{\prime} \in Z(A)$.

LEMMA 3.11. Let $f=\psi_{i}^{-1}\left(f^{\prime}\right)$ and $g=\psi_{i}^{-1}\left(g^{\prime}\right)$. Then $f, g$ are orthogonal idempotents in the center of $A$ which sum to 1. They are independent of the defining $i$. Moreover, $\psi_{i}$ is an isomorphism from $f A$ onto $f^{\prime} A$, and a anti-isomorphism from $g A$ onto $g^{\prime} A$, so that $\psi_{i} \in$ GAut $A$.

Proof. That $f, g$ are idempotents summing to 1 follows simply from the fact that $\psi_{i}$ is an automorphism relative to the $\circ$-product. Orthogonality is shown as it was for $f^{\prime}, g^{\prime}$ above. To prove the remaining statements we observe that from $\psi\left(a b e_{i j}\right)=$ $2 \psi\left(a e_{i i}\right) \circ \psi\left(b e_{i j}\right)$ we can establish: $\psi_{i}(a b) \alpha_{i j}=\psi_{i}(a) \psi_{i}(b) \alpha_{i j}$ and $\beta_{j i} \psi_{i}(a b)=$ $\beta_{j i} \psi_{i}(b) \psi_{i}(a)$. Multiplication by $\alpha_{j i}$ and $\beta_{i j}$ respectively demonstrates

$$
\begin{aligned}
& \psi_{i}(a b) f^{\prime}=\psi_{i}(a) \psi_{i}(b) f^{\prime}, \\
& g^{\prime} \psi_{i}(a b)=g^{\prime} \psi_{i}(b) \psi_{i}(a) .
\end{aligned}
$$

But then equation (3.19) with $a=f$ shows $g^{\prime} \psi_{i}(f b)=g^{\prime} \psi_{i}(b) f^{\prime}=g^{\prime} f^{\prime} \psi_{i}(b)=0$. Hence, $\psi_{i}$ maps $f A$ into $f^{\prime} A$, and it is an isomorphism on $f A$ according to (3.18). Likewise $\psi_{i}$ is an anti-isomorphism of $g A$ into $g^{\prime} A$. We use the fact that $A=f A+$ $g A$, and the fact that $\psi_{i}$ is onto to conclude it is onto the components $f A$ and $g A$.

What remains to be shown is that $f, g \in Z(A)$ and $f, g$ are independent of $i$. We note that $\psi_{i}(a f)=\psi_{i}(a f) f^{\prime}$, and $\psi_{i}(f a)=\psi_{i}(f a) f^{\prime}$ since $f^{\prime}$ is the identity on $f^{\prime} A$. 
Therefore (3.18) shows that $\psi_{i}(a f)=\psi_{i}(a) f^{\prime}$ and also $\psi_{i}(f a)=f^{\prime} \psi_{i}(a)=\psi_{i}(a) f^{\prime}$. Hence $\psi_{i}(a f)=\psi_{i}(f a)$ for all $a$, and $a f=f a$. To prove that $f$ lies in the nucleus of $A$, it suffices to show $f$ acts as the identity on $f A$ and $f A \cdot g A=0$. But this can be accomplished by using (3.18) with af instead of $a$, and $f$ instead of $b$, and by using (3.19) with $a f$ in place of $a$, and $b g$ in place of $b$. Thus, $f \in Z(A)$ and $g=1-f \in$ $Z(A)$ also.

Now relations (3.11) and (3.13) demonstrate

$$
\begin{aligned}
& \alpha_{i j} \psi_{j}(a)=\psi_{i}(a) \alpha_{i j}, \\
& \psi_{j}(a) \beta_{j i}=\beta_{j i} \psi_{i}(a) .
\end{aligned}
$$

These equations show that $f^{\prime} \psi_{j}(a)=\alpha_{j i} \psi_{i}(a) \alpha_{i j}$ and $\psi_{j}(a) g^{\prime}=\beta_{j i} \psi_{i}(a) \beta_{i j}$. Substituting $a=f$ we see $f^{\prime} \psi_{j}(f)=f^{\prime}$ and $\psi_{j}(f) g^{\prime}=0$. Thus $\psi_{j}(f)=f^{\prime}$ and we are done.

LEMMA 3.12. There is an invertible diagonal matrix $v$ in $M_{n}(N)$ such that $\chi_{v} \psi=\psi_{1}^{\#}$.

Proof. It is an easy consequence of (3.8) that $\beta_{j i} f^{\prime} A=f^{\prime} A \beta_{j i}=g^{\prime} A \alpha_{i j}=\alpha_{i j} g^{\prime} A$ $=0$ for all $i \neq j$, and from the remarks immediately following equations (3.20) and (3.21), it follows that $\psi_{j}(f a)=f^{\prime} \psi_{j}(f a)=\alpha_{j 1} \psi_{1}(f a) \alpha_{i j}$ and $\psi_{j}(g a)=g^{\prime} \psi_{j}(g a)=$ $\beta_{j 1} \psi_{1}(g a) \beta_{1 j}$. Therefore writing $a=f a+g a$, we conclude from these observations that

$$
\psi_{j}(a)=\left(\alpha_{j 1}+\beta_{j 1}\right) \psi_{1}(a)\left(\alpha_{1 j}+\beta_{1 j}\right) \text { for all } a .
$$

Let $v$ denote the matrix with 1 in the $(1,1)$ position, $\alpha_{j 1}+\beta_{j 1}$ in the $(j, j)$ slot for $j \geqslant 2$, and 0 elsewhere. Then $v^{-1}$ is also diagonal with diagonal entries 1 , $\alpha_{12}+\beta_{12}, \ldots, \alpha_{1 n}+\beta_{1 n}$. Thus, $\chi_{v} \psi\left(a e_{j j}\right)=v^{-1} \psi_{j}(a) e_{j j} v=\psi_{1}(a) e_{i j}$. Calculation using (3.8), (3.10) and (3.22) shows

$$
\begin{aligned}
\chi_{v} \psi\left(a e_{i j}\right)= & \left(\alpha_{1 i}+\beta_{1 i}\right)\left(\alpha_{i 1}+\beta_{i 1}\right) \psi_{1}(a)\left(\alpha_{1 i}+\beta_{1 i}\right) \alpha_{i j}\left(\alpha_{j 1}+\beta_{j 1}\right) e_{i j} \\
& +\left(\alpha_{1 j}+\beta_{1 j}\right) \beta_{j i}\left(\alpha_{i 1}+\beta_{i 1}\right) \psi_{1}(a)\left(\alpha_{1 i}+\beta_{1 i}\right)\left(\alpha_{i 1}+\beta_{i 1}\right) e_{j i} \\
= & \psi_{1}(a) \alpha_{1 j} \alpha_{j 1} e_{i j}+\beta_{1 i} \beta_{i 1} \psi_{1}(a) e_{j i} \\
= & \psi_{1}(a) f^{\prime} e_{i j}+g^{\prime} \psi_{1}(a) e_{j i} \\
= & \psi_{1}(f a) e_{i j}+\psi_{1}(g a) e_{j i} .
\end{aligned}
$$

Hence, $\chi_{v} \psi=\psi_{1}^{\#} \in(\text { GAut } A)^{\#}$, and this concludes the proof of Lemma 3.12, and Theorem 3.6.

As a consequence of this theorem and Corollary 3.5 we have

THEOREM 3.13. Let $A$ be an arbitrary nonassociative algebra with 1 such that the nucleus $N$ of $A$ is Artinian. If char $\Phi \neq 2$, then

$$
\text { GAut } M_{n}(A)=\mathrm{GL}(n, N) \cdot(\text { GAut } A)^{\#} \text {. }
$$

REMARK. It should be noted that the subgroup $\mathrm{GL}(n, N)$ of conjugations is a normal subgroup of GAut $M_{n}(A)$, hence of Aut $M_{n}(A)$ and of AAut $M_{n}(A)$ as well, 
and it equals

$$
\text { GAut }_{A} M_{n}(A)=\left\{\varphi \in \text { GAut } M_{n}(A) \mid \varphi(a I)=a I \text { for all } a \in A\right\}
$$

if $Z(A)=N$.

COROLlaRY 3.14. With assumptions as in Theorem 3.13,

$$
\text { Aut } M_{n}(A)=\mathrm{GL}(n, N) \cdot(\text { Aut } A)^{\#}
$$

and

$$
\text { AAut } M_{n}(A)=\mathrm{GL}(n, N) \cdot(\text { AAut } A)^{\#} \text {, }
$$

so that $M_{n}(A)$ has antiautomorphisms if and only if $A$ does.

Proposition 3.15. If $N$ is Artinian, Aut $M_{n}(A)$ has finite index in GAut $M_{n}(A)$.

Proof. Since $N$ is Artinian with 1, we can find orthogonal central idempotents $e_{1}, \ldots, e_{q}$ such that $1=e_{1}+\cdots+e_{q}$ and each $e_{i}$ cannot be expressed as the sum of two other central idempotents. Then $M_{n}(N)=\bigoplus \sum_{i=1}^{q} S_{i}$ where $S_{i}=e_{i} I$. $M_{n}(N)$ is an indecomposable ideal of $M_{n}(N)$. For any ideal $T$ of $M_{n}(N), T=$ $\bigoplus \sum_{i=1}^{q} e_{i} I \cdot T$, so if $T$ is indecomposable, $T=S_{j}$ for some $j$. Given $\varphi \in$ GAut $M_{n}(A)$ with idempotents $f I, g I, f^{\prime} I, g^{\prime} I$, then $f e_{i}+g e_{i}=e_{i}=f^{\prime} e_{i}+g^{\prime} e_{i}$ implies $f I \cdot M_{n}(N), g I \cdot M_{n}(N), f^{\prime} I \cdot M_{n}(N), g^{\prime} I \cdot M_{n}(N)$ are all just the sums of certain of the $S_{i}$. We conclude that each $\varphi\left(S_{i}\right)$ is an indecomposable ideal of $M_{n}(N)$ and so equals some $S_{j}$. Thus, $\varphi$ permutes the summands and the subgroup of generalized automorphisms fixing all the $S_{i}$ has index at most $q$ ! in GAut $M_{n}(A)$. Since every $\psi$ in that subgroup acts as an automorphism or antiautomorphism on each $S_{i}$, the index of Aut $M_{n}(A)$ in GAut $M_{n}(A)$ is at most $q ! 2^{q}$.

The next example demonstrates that Corollary 3.14 is false for $N$ arbitrary.

Let $\Phi[t]$ denote the ring of polynomials over $\Phi$, and let $B$ be the ideal of $\Phi[t]$ generated by $t^{2}+1$. Define $A$ to be $\{(a, b) \mid a, b \in \Phi[t]$ and $a \equiv b \bmod B\}$. If $w$ denotes the matrix

$$
\left(\begin{array}{cc}
(1,-t) & \left(0,1+t^{2}\right) \\
(t, 1) & (1,-t)
\end{array}\right),
$$

then $w$ lies in $M_{2}(\Phi[t] \times \Phi[t])$ but not in $M_{2}(A)$. The matrix $w$ is invertible, and conjugation by $w$ leaves $M_{2}(A)$ invariant. If $\chi_{w}=\chi_{v} \theta^{\#}$ for $v \in M_{2}(A)$, and $\theta^{\#} \in(\text { Aut } A)^{\#}$, then for each $c \in A, c I=\chi_{w}(c I)=\chi_{v} \theta^{\#}(c I)=\theta(c) I$, which implies $\theta=1$. Now if $\chi_{w}=\chi_{v}$, then

$$
w=\left(\begin{array}{cc}
(r, s) & 0 \\
0 & (r, s)
\end{array}\right) v
$$

where $r, s$ are nonzero elements of $\Phi(t)$. The determinant of $v$ must be a unit in $A$, so it has the form $(\alpha, \alpha)$ where $\alpha \in \Phi$. From taking determinants we conclude $(1,-1)=\left(r^{2}, s^{2}\right)(\alpha, \alpha)$, and hence both $r$ and $s$ are in $\Phi$. We write

$$
v=\left(\begin{array}{ll}
\left(u_{1}, v_{1}\right) & \left(u_{2}, v_{2}\right) \\
\left(u_{3}, v_{3}\right) & \left(u_{4}, v_{4}\right)
\end{array}\right)
$$


and compute

$$
w=\left(\begin{array}{cc}
(r, s) & 0 \\
0 & (r, s)
\end{array}\right) v .
$$

Then $1=r u_{1}$ where $r \in \Phi$, and since $\left(u_{1}, v_{1}\right) \in A$, it follows $v_{1}=r^{-1}$ also. But then $-t=s v_{1}=s r^{-1} \in \Phi$, a contradiction. Consequently $\chi_{w} \in$ Aut $M_{2}(A)$, and $\chi_{w} \notin \mathrm{GL}(2, A) \cdot(\text { Aut } A)^{\#}$.

REMARK. We are indebted to L. Levy for his assistance in the creation of this example and for making us aware of work by Rosenberg and Zelinsky [10] on this subject.

4. $M_{n}(A)$ under the Lie product. Let $L_{n}(A)$ denote the anticommutative algebra obtained by taking $M_{n}(A)$ under the product $[x y]=x y-y x$, and let $L_{n}^{\prime}(A)=$ $\left[L_{n}(A), L_{n}(A)\right]$. The algebra $L_{n}^{\prime}(A)$ is an ideal of $L_{n}(A)$ which is invariant under all automorphisms and derivations of $L_{n}(A)$. It is spanned by the elements

$$
\begin{aligned}
a e_{i j} & =\left[a e_{i i}, e_{i j}\right], \quad i \neq j, \\
a b e_{i i}-b a e_{j j} & =\left[a e_{i j}, b e_{j i}\right] .
\end{aligned}
$$

Two noteworthy cases of the second equation occur when $i=j$ and when $b=1$. The elements obtained are $[a b] e_{i i}$ and $a e_{i i}-a e_{j i}$. These elements in fact generate all of the ones of the second type since $a b e_{i i}-b a e_{i j}=[a b] e_{i i}+b a e_{i i}-b a e_{i j}$. Each element in $L_{n}^{\prime}(A)$ has trace in $[A, A]$. Conversely if $x=\sum_{i=1}^{n} a_{i i} e_{i i}$ where $t=$ $\sum_{i=1}^{n} a_{i i} \in[A, A]$, then $x=t e_{n n}+\sum_{i=1}^{n-1} a_{i i} e_{i i}-a_{i i} e_{n n} \in L_{n}^{\prime}(A)$. Thus $L_{n}^{\prime}(A)$ is precisely the space of all elements with trace in $[A, A]$. Using the above products one can also verify that if $n \geqslant 3$ when $\operatorname{char} \Phi=2$, or if $n \geqslant 2$ when char $\Phi \neq 2$, then $L_{n}^{\prime}(A)=\left[L_{n}^{\prime}(A), L_{n}^{\prime}(A)\right]$.

Let $Z$ denote the center of $L_{n}(A)$; that is $Z=\left\{z \in L_{n}(A) \| z, x\right]=0$ for all $\left.x \in L_{n}(A)\right\}$. If $C(A)=\{c \in A \mid c a=a c$ for all $a \in A\}$, then calculation shows that $Z=\{c I \mid c \in C(A)\}$. The center is an ideal of $L_{n}(A)$ invariant under automorphisms and derivations of $L_{n}(A)$. Its intersection with $L_{n}^{\prime}(A)$ is the center of $L_{n}^{\prime}(A)$ and is also invariant. We define $K_{n}(A)=L_{n}^{\prime}(A) / Z \cap L_{n}^{\prime}(A)$. Our goal in this section will be to investigate the derivation algebras of $M_{n}(A), L_{n}(A), L_{n}^{\prime}(A)$, and $K_{n}(A)$, and the automorphism groups of the last three. Each derivation (automorphism) of $M_{n}(A)$ induces a derivation (automorphism) of $L_{n}(A)$. This assertion remains true for the pairs $L_{n}(A), L_{n}^{\prime}(A)$ and $L_{n}^{\prime}(A), K_{n}(A)$. So we have the natural homomorphisms

$$
\operatorname{Der} M_{n}(A) \stackrel{\rho}{\rightarrow} \operatorname{Der} L_{n}(A) \stackrel{\sigma}{\rightarrow} \operatorname{Der} L_{n}^{\prime}(A) \stackrel{\tau}{\rightarrow} \operatorname{Der} K_{n}(A) .
$$

Lemma 4.1. Suppose $n \geqslant 3$ if $\operatorname{char} \Phi=2$, or $n \geqslant 2$ if $\operatorname{char} \Phi \neq 2$. Then

(a) $\sigma \rho, \rho$, and $\tau$ are one-to-one, and

(b) the kernel of $\sigma$ consists of all Lie homomorphisms of $L_{n}(A)$ into $Z$, denoted by $\operatorname{LHom}\left(L_{n}(A), Z\right)$.

Proof. If $\partial$ is in the kernel of $\tau$, then $\partial$ maps $L_{n}^{\prime}(A)$ into $Z$. But then $L_{n}^{\prime}(A)=\left[L_{n}^{\prime}(A), L_{n}^{\prime}(A)\right]$ is sent to 0 , which shows $\partial=0$. Since $L_{n}(A)=M_{n}(A)$ as vector spaces, the kernel of $\rho$ must be 0 . Now any derivation $\partial$ in the kernel of $\sigma \rho$ 
must be 0 on the elements $a e_{i j}$ for $i \neq j$. Since these elements generate $M_{n}(A)$ under the usual matrix product, it follows that $\partial$ is identically 0 . Turning our attention to part (b), we assume $\partial$ is in the kernel of $\sigma$. Then $\partial$ is 0 on the elements in (4.1) so we need only examine the effect of $\partial$ on elements of the form $a e_{i j}$. For $k \neq l$ we have $\left[a e_{j j} b e_{k l}\right]=\delta_{j k} a b e_{j l}-\delta_{j l} b a e_{k j}$, and $\partial$ applied to this relation shows $\left[\partial\left(a e_{j j}\right), b e_{k l}\right]=0$ for all $b \in A$. From this we deduce $\partial\left(a e_{j j}\right) \in Z$. Any Lie homomorphism of $L_{n}(A)$ into $Z$ is a derivation of $L_{n}(A)$ which necessarily vanishes on $L_{n}^{\prime}(A)$. Thus the kernel of $\sigma$ is $\operatorname{LHom}\left(L_{n}(A), Z\right)$ as claimed.

In a similar fashion we have the natural homomorphisms

$$
\text { Aut } M_{n}(A) \stackrel{\rho}{\rightarrow} \text { Aut } L_{n}(A) \stackrel{\sigma}{\rightarrow} \text { Aut } L_{n}^{\prime}(A) \stackrel{\tau}{\rightarrow} \text { Aut } K_{n}(A) \text {. }
$$

LemMA 4.2. Assume $n \geqslant 3$ if $\operatorname{char} \Phi=2$, or $n \geqslant 2$ if char $\Phi \neq 2$. Then

(a) $\sigma \rho, \rho$, and $\tau$ are one-to-one, and

(b) the kernel of $\sigma$ consists of all mappings $\varphi$ such that $\varphi$ is the identity map plus an element in $\operatorname{LHom}\left(L_{n}(A), Z\right)$.

Since the proof of 4.2 involves only a slight modification of the proof of 4.1 , we omit it.

The Lie nucleus, which we introduce next, will play the role that $M_{n}(N)$ played in our investigations of GAut $M_{n}(A)$.

For each anticommutative algebra $L$ with product [ ] the Lie nucleus of $L$ is

$$
\nu(L)=\{x \in L \mid[[x y] z]+[[y z] x]+[[z x] y]=0 \text { for all } y, z \in L\} .
$$

If $\operatorname{ad}_{x}$ denotes the map $\operatorname{ad}_{x}(y)=[x y]$, then an alternate description of the Lie nucleus is

$$
\nu(L)=\left\{x \in L \mid \operatorname{ad}_{x} \in \operatorname{Der} L\right\} .
$$

The space $\nu(L)$ is invariant under automorphisms and derivations of $L$. As a result, if $x, x^{\prime} \in \nu(L)$ then $\operatorname{ad}_{x}\left(x^{\prime}\right) \in \nu(L)$, and $\nu(L)$ is a subalgebra of $L$. It follows then that $\nu(L)$ is a Lie algebra.

Let us specialize now to the case $L=L_{n}(A)$. We define $D(A)=\left\{a \in A \mid \operatorname{ad}_{a} \in\right.$ Der $A\}$, and note that this amounts to saying $D(A)=\{a \in A \|[a, b c]=[a b] c+$ $b[a c]\}$.

Lemma 4.3. Assume $n \geqslant 3$. Then $\nu\left(L_{n}(A)\right)=L_{n}(N)+D(A) I$.

Proof. Using the definition of $D(A) I$ above and the fact that $M_{n}(N)$ is the nucleus of $M_{n}(A)$, one can show that $L_{n}(N)+D(A) I \subseteq \nu\left(L_{n}(A)\right)$. For the proof of the reverse containment let

$$
\lambda(x, y, z)=[[x y] z]+[[y z] x]+[[z x] y]
$$

and assume $x=\sum b_{i j} e_{i j} \in \nu\left(L_{n}(A)\right)$. Then since $\nu\left(L_{n}(A)\right)$ is a subalgebra containing $L_{n}(N)$, for $k, l, m$ distinct we have $\left[\left[\left[x e_{l k}\right] e_{m k}\right] e_{l m}\right]=b_{k l} e_{l k} \in \nu\left(L_{n}(A)\right)$. Dropping the subscript on $b_{k l}$ for simplicity, we have $b e_{l k} \in \nu\left(L_{n}(A)\right)$ for $l \neq k$. From $\lambda\left(b e_{l k}, a e_{k m}, c e_{m k}\right)=0$ for all $a, c \in A$ we obtain $(b a) c e_{l k}-b(a c) e_{l k}=0$, and hence $(b a) c=b(a c)$. Similarly $\lambda\left(a e_{m l}, b e_{l k}, c e_{k l}\right)=0$ and $\lambda\left(a e_{m k}, c e_{k l}, b e_{l k}\right)=0$ 
imply $(a b) c=a(b c)$ and $(a c) b=a(c b)$. We conclude $b$ is in $N$, and $b e_{l k} \in L_{n}(N)$ $\subseteq \nu\left(L_{n}(A)\right)$.

It suffices to suppose $x=\sum_{i=1}^{n} b_{i} e_{i i} \in \nu\left(L_{n}(A)\right)$. For $k \neq l$ we have

$$
\left[x, e_{k l}\right]=\left(b_{k}-b_{l}\right) e_{k l} \in \nu\left(L_{n}(A)\right)
$$

and by the above $b_{k}-b_{l} \in N$. Now a computation of $\lambda\left(x, a e_{q r}, c e_{s t}\right)$ shows

$$
\begin{aligned}
\delta_{r s}\left(b_{q} a-\right. & \left.a b_{r}\right) c e_{q t}-\delta_{t q} c\left(b_{q} a-a b_{r}\right) e_{s r} \\
& +\delta_{r s}\left\{(a c) b_{t}-b_{q}(a c)\right\} e_{q t}-\delta_{t q}\left\{b_{s}(c a)-(c a) b_{r}\right\} e_{s r} \\
& -\delta_{r s} a\left(c b_{t}-b_{s} c\right) e_{q t}+\delta_{t q}\left(c b_{t}-b_{s} c\right) a e_{s r}=0 .
\end{aligned}
$$

This expression can be made more transparent by writing for each $i$ and $j$ $b_{i}=b_{q}+\beta_{i}, b_{j}=b_{r}+\gamma_{j}$ where $\beta_{i}, \gamma_{j} \in N$. The result is

$$
\begin{aligned}
0= & \delta_{r s}\left\{\left[b_{q} a\right] c+\left[a c, b_{q}\right]+a\left[c b_{q}\right]+\left(a \beta_{s}\right) c-a\left(\beta_{r} c\right)\right\} e_{q t} \\
& -\delta_{q t}\left\{c\left[b_{r} a\right]+\left[c a, b_{r}\right]-\left[c b_{r}\right] a+\left(c \gamma_{q}\right) a-c\left(\gamma_{t} a\right)\right\} e_{s r} .
\end{aligned}
$$

This proves that $b_{q} \in D(A)$ for each $q$ and hence, $x=\sum_{i=1}^{n-1}\left(b_{i}-b_{n}\right) e_{i i}+b_{n} I \in$ $L_{n}(N)+D(A) I$. Thus, the calculation shows $\nu\left(L_{n}(A)\right) \subseteq L_{n}(N)+D(A) I$, and it also demonstrates that $D(A) I \subseteq \nu\left(L_{n}(A)\right)$ as was asserted above.

The same argument can be used to show

LemMA 4.4. For $n \geqslant 3, \nu\left(L_{n}^{\prime}(A)\right)=\nu\left(L_{n}(A)\right) \cap L_{n}^{\prime}(A)$ and $\nu\left(K_{n}(A)\right)=\nu\left(L_{n}^{\prime}(A)\right)$ $+Z \cap L_{n}^{\prime}(A)$.

Let us adopt the notation $Z^{\prime}=Z \cap L_{n}^{\prime}(A)$ so that $K_{n}(A)=L_{n}^{\prime}(A) / Z^{\prime}$.

LemMA 4.5. Assume char $\Phi \neq 2,3$, and let $T$ be a transformation on $K_{n}(A)$ with the property:

$$
\left[e_{i i}-e_{j j}+Z^{\prime}, T\left(x+Z^{\prime}\right)\right]=T\left(\left[e_{i i}-e_{i j}+Z^{\prime}, x+Z^{\prime}\right]\right)
$$

for all $x \in L_{n}^{\prime}(A)$ and all $i, j$. Then for each pair $i \neq j, T$ induces a transformation $T_{i j}$ on $A$ such that

$$
T\left(a e_{i j}+Z^{\prime}\right)=T_{i j}(a) e_{i j}+Z^{\prime}
$$

Proof. When char $\Phi \neq 2,3$ the space $A e_{i j}+Z^{\prime}$ can be characterized in the following way:

$$
A e_{i j}+Z^{\prime}=\left\{x+Z^{\prime} \in K_{n}(A) \mid\left[e_{i i}-e_{j j}+Z^{\prime}, x+Z^{\prime}\right]=2 x+Z^{\prime}\right\} .
$$

The definition of $T$ makes this space $T$-invariant. Thus, for each $a \in A$, there is a unique $b \in A$ such that the coset $b e_{i j}+Z^{\prime}$ equals $T\left(a e_{i j}+Z^{\prime}\right)$. The $T_{i j}$ desired is given by $T_{i j}(a)=b$.

Lemma 4.5 will be used next to study derivations $\partial$ with the property that $\partial\left(e_{i i}-e_{j j}+Z^{\prime}\right)=0$, and in the future to investigate automorphisms $\varphi$ such that $\varphi\left(e_{i i}-e_{j j}+Z^{\prime}\right)=e_{i i}-e_{j j}+Z^{\prime}$.

Theorem 4.6. Assume $n \geqslant 3$ and char $\Phi \neq 2$, 3. Let $\partial \in \operatorname{Der} K_{n}(A)$ be such that $\partial\left(e_{i i}-e_{j j}+Z^{\prime}\right)=0$ for all $i, j$. Then there is $a \Delta \in(\operatorname{Der} A)^{\#}$ and a diagonal matrix $y \in M_{n}(N)$ such that $\partial=\Delta+\operatorname{ad}_{y}$. 
Proof. The derivation $\partial$ satisfies the hypotheses of Lemma 4.5, and so

$$
\partial\left(e_{i j}+Z^{\prime}\right)=\alpha_{i j} e_{i j}+Z^{\prime} \text { for } i \neq j \text {. }
$$

The elements $\alpha_{i j}$ belong to $N$ since $e_{i j}+Z$ is in $\nu\left(K_{n}(A)\right)$ which is derivation-invariant, and off-diagonal entries of $\nu\left(K_{n}(A)\right)$ lie in $N$.

Applying $\partial$ to the following relations

$$
\begin{aligned}
{\left[e_{i k}+Z^{\prime}, e_{k j}+Z^{\prime}\right] } & =e_{i j}+Z^{\prime} \text { for } i, j, k \text { distinct, } \\
{\left[e_{i j}+Z^{\prime}, e_{j i}+Z^{\prime}\right] } & =e_{i i}-e_{j j}+Z^{\prime}
\end{aligned}
$$

shows that

$$
\alpha_{i k}+\alpha_{k j}=\alpha_{i j}, \quad \alpha_{i j}+\alpha_{j i}=0 .
$$

Let $y$ be the diagonal matrix with $0, \alpha_{21}, \ldots, \alpha_{n 1}$ down the diagonal. Then $\operatorname{ad}_{y}\left(e_{i j}+Z^{\prime}\right)=\left(\alpha_{i 1}-\alpha_{j 1}\right) e_{i j}+Z^{\prime}=\alpha_{i j} e_{i j}+Z^{\prime}$. Thus $\Delta=\partial-\operatorname{ad}_{y} \in \operatorname{Der} K_{n}(A)$ has the property that $\Delta\left(e_{i j}+Z^{\prime}\right)=0$ as well as $\Delta\left(e_{i i}-e_{j j}+Z^{\prime}\right)=0$ for each pair $i, j$ with $i \neq j$. According to Lemma 4.5, $\Delta$ induces transformations $\Delta_{i j}$ of $A$ such that $\Delta\left(a e_{i j}+Z^{\prime}\right)=\Delta_{i j}(a) e_{i j}+Z^{\prime}$ for $i \neq j$. Since

$$
\Delta\left(a e_{i j}+Z^{\prime}\right)=\left[\Delta\left(a e_{i k}+Z^{\prime}\right), e_{k j}+Z^{\prime}\right]=\Delta_{i k}(a) e_{i k}+Z^{\prime},
$$

it follows that $\Delta_{i j}=\Delta_{i k}$ for $k \neq j$. An analogous argument shows the first subscript can also be altered. Thus, all the induced transformations are equal and we call the common map $\eta$.

By applying $\Delta$ to the relation

$$
\left[a e_{i k}+Z^{\prime}, b e_{k j}+Z^{\prime}\right]=a b e_{i j}+Z^{\prime} \text { for } i, k, j \text { distinct }
$$

we obtain $\eta \in \operatorname{Der} A$. Thus, at least on elements of the form $a e_{i j}+Z^{\prime}, \Delta=\eta^{\#}$, the derivation obtained by acting by $\eta$ on each entry. But these elements generate $K_{n}(A)$ as the next two relations show:

$$
\begin{gathered}
{\left[a e_{i j}+Z^{\prime}, e_{j i}+Z^{\prime}\right]=a e_{i i}-a e_{j j}+Z^{\prime},} \\
{\left[a e_{i i}-a e_{j j}+Z^{\prime}, b e_{i i}-b e_{k k}+Z^{\prime}\right]=[a b] e_{i i}+Z^{\prime} .}
\end{gathered}
$$

And these equations can be used to verify that $\Delta=\eta^{\#} \in(\operatorname{Der} A)^{\#}$.

THEOREM 4.7. Let $\partial$ be an arbitrary element of Der $K_{n}(A)$ where char $\Phi \neq 2,3$ and $n \geqslant 3$. Then there is a $w \in M_{n}(N)$ such that $\partial^{\prime}=\partial-a_{w}$ has the property that $\partial^{\prime}\left(e_{i i}-e_{j j}+Z^{\prime}\right)=0$ for all $i, j$.

Proof. When $i \neq j$,

$$
\partial\left[e_{i i}-e_{j j}+Z^{\prime}\right]=\left[\partial\left(e_{i j}+Z^{\prime}\right), e_{j i}+Z^{\prime}\right]+\left[e_{i j}+Z^{\prime}, \partial\left(e_{j i}+Z^{\prime}\right)\right],
$$

and from this relation it is apparent that $\partial\left(e_{i i}-e_{j j}+Z^{\prime}\right)$ has a unique coset representative with nonzero entries only in the $i$ th and $j$ th rows and columns. When $j=n$ let us denote this coset in the following fashion:

$$
\begin{aligned}
\partial\left(e_{i i}-e_{n n}+Z^{\prime}\right)= & \sum_{k \neq i} \beta_{k i}^{i} e_{k i}+\sum_{l} \beta_{i l}^{i} e_{i l} \\
& +\sum_{\substack{k \neq i \\
k \neq n}} \beta_{k n}^{i} e_{k n}+\sum_{l \neq i} \beta_{n l}^{i} e_{n l}+Z^{\prime}
\end{aligned}
$$


Since $\partial\left(e_{i i}-e_{j j}+Z^{\prime}\right)=\partial\left(e_{i i}-e_{n n}+Z^{\prime}\right)-\partial\left(e_{j j}-e_{n n}+Z^{\prime}\right)$, we have

$$
\beta_{k n}^{i}=\beta_{k n}^{j}, \beta_{n k}^{i}=\beta_{n k}^{j}, \text { for } k \text { distinct from } i, j \text {, and } n \text {. }
$$

The elements $\beta_{k l}^{i}$ lie in $N$ for $k \neq l$ since the Lie nucleus is preserved by derivations. Showing that the diagonal $\beta$ 's are 0 , and deriving relationships between the various $\beta$ 's for different $i$ 's, such as (4.8) above, will enable us to construct the matrix $w$.

Let $\partial\left(e_{i j}+Z^{\prime}\right)=\sum \alpha_{k l} e_{k l}+Z^{\prime}$, and compare coefficients of the $(i, i)$ and $(i, j)$ entries in

$$
\left[\partial\left(e_{i i}-e_{n n}+Z^{\prime}\right), e_{i j}+Z^{\prime}\right]+\left[e_{i i}-e_{n n}+Z^{\prime}, \partial\left(e_{i j}+Z^{\prime}\right)\right]=\partial\left(e_{i j}+Z^{\prime}\right) .
$$

The resulting relations are

$$
-\beta_{j i}^{i}=\alpha_{i i}, \quad-\beta_{i i}^{i}+\alpha_{i j}=\alpha_{i j}
$$

Now if $e_{i i}-e_{n n}$ is replaced by $e_{j j}-e_{n n}$ in (4.9) an analogous argument shows

$$
-\beta_{j i}^{j}=-\alpha_{i i} \text {. }
$$

Therefore it follows that

$$
\beta_{i i}^{i}=0 \text { and } \beta_{j i}^{i}=-\beta_{j i}^{j} \text {. }
$$

Finally, if $e_{i n}$ is used in place of $e_{i j}$ in (4.9), one can show $\beta_{n n}^{i}=0$ also. We define

$$
\begin{aligned}
\gamma_{j i} & =\beta_{j i}^{i} \text { for } j, i \neq n, \\
\gamma_{k n} & =-\beta_{k n}^{j} \text { for } j \neq k, n, \\
\gamma_{n k} & =\beta_{n k}^{j} .
\end{aligned}
$$

The equations in (4.8) show that $\gamma_{k n}$ and $\gamma_{n k}$ are independent of the defining $j$. Let $w=\sum \gamma_{k l} e_{k l}$ and note that $w \in M_{n}(N)$, so that $\operatorname{ad}_{w}$ is a derivation. The image of each $e_{i i}-e_{n n}+Z^{\prime}$ under $\operatorname{ad}_{w}$ is the same as under $\partial$ except for the $(i, n)$ and $(n, i)$ entries. Thus, if $\partial^{\prime}=\partial-\operatorname{ad}_{w}$, then $\partial^{\prime}\left(e_{i i}-e_{n n}+Z^{\prime}\right)=\xi_{i n} e_{i n}+\xi_{n i} e_{n i}+Z^{\prime}$. Comparison of the $(n, j)$ and $(i, n)$ coefficients in (4.9) now shows

$$
\xi_{n i}-\alpha_{n j}=\alpha_{n j}, \quad 2 \alpha_{i n}=\alpha_{i n} .
$$

Similar equations resulting from the use of $e_{j j}-e_{n n}$ are

$$
-\xi_{j n}+\alpha_{i n}=-\alpha_{i n}, \quad-2 \alpha_{n j}=-\alpha_{n j} \text {. }
$$

Thus, $\xi_{j n}=0$ and $\xi_{n i}=0$ for each $i$ and $j$, and $\partial^{\prime}=\partial-\operatorname{ad}_{w}$ is the desired derivation.

Combining Theorems 4.6 and 4.7 gives

ThEOREM 4.8. Assume char $\Phi \neq 2,3$ and $n \geqslant 3$. Then for any algebra $A$ with 1 , $\operatorname{Der} K_{n}(A)=(\operatorname{Der} A)^{\#}+\operatorname{ad}_{M_{n}(N)}$.

COROLlaRY 4.9. With assumptions as in the previous theorem,

$$
\operatorname{Der} M_{n}(A)=\operatorname{Der} L_{n}^{\prime}(A)=\operatorname{Der} K_{n}(A)=(\operatorname{Der} A)^{\#}+\operatorname{ad}_{M_{n}(N)}
$$


Proof. Each element of Der $K_{n}(A)$ is a derivation of $M_{n}(A)$ and $L_{n}^{\prime}(A)$. However the mappings $\tau \sigma \rho$ and $\tau$ in Lemma 4.1 are the canonical injections of $\operatorname{Der} M_{n}(A)$ and Der $L_{n}^{\prime}(A)$ into Der $K_{n}(A)$. So these algebras can be no larger than $\operatorname{Der} K_{n}(A)$, and the above equalities hold.

Using 4.1 and this result we have

Corollary 4.10. Der $L_{n}(A)=(\operatorname{Der} A)^{\#}+\operatorname{ad}_{M_{n}(N)}+\operatorname{LHom}\left(L_{n}(A), Z\right)$.

REMARKS. Martindale [8] has obtained a result in a similar vein as Corollary 4.10. He showed that if $R$ is a primitive associative ring with a nontrivial idempotent, then any Lie derivation of $R$ is the sum of a derivation of $R$ plus a Lie homomorphism of $R$ into $Z$.

It should be commented that in showing $\operatorname{Der} M_{n}(A)=(\operatorname{Der} A)^{\#}+\operatorname{ad}_{M_{n}(N)}$ no restrictions on char $\Phi$ or on $n$ are necessary. In this case $A e_{i j}=\left\{x \in M_{n}(A) \mid e_{i i} x=\right.$ $\left.x e_{i j}=x\right\}$ so that any derivation $\partial$ with the property that $\partial\left(e_{i i}\right)=0$ induces a transformation $\partial_{i j}$ on $A e_{i j}$ regardless of char $\Phi$. The additional hypotheses are needed in the computation of $\operatorname{Der} K_{n}(A)$ to determine the structure of the Lie nucleus. But in the calculation of $\operatorname{Der} M_{n}(A), M_{n}(N)$ is mapped into itself, so no such contortions are necessary. Rather than make a separate case for $M_{n}(A)$, we chose to treat $M_{n}(A), L_{n}(A), L_{n}^{\prime}(A)$, and $K_{n}(A)$ simultaneously at this small expense.

Corollary 4.11 (Jacobson [3, Theorem 8, p. 215]). Let $S$ be a finite dimensional central simple associative algebra over $\Phi$. Then every derivation of $S$ is inner.

Proof. Let $F$ be a splitting field for $S$ over $\Phi$. Then $S \otimes_{\Phi} F \approx M_{n}(F)$. Thus by Corollary 4.9 and the preceding remarks, $\operatorname{Der}_{F}\left(S \otimes_{\Phi} F\right) \approx \operatorname{Der}_{F}\left(M_{n}(F)\right) \approx$ $\operatorname{ad}_{M_{n}(F)}$. Now if $\partial \in \operatorname{Der} S$, then $\partial \otimes 1 \in \operatorname{Der}_{F}\left(S \otimes_{\Phi} F\right)$. In particular, for each $x \in S, \operatorname{ad}_{x} \in \operatorname{Der} S$, and since $S$ is central it follows that $\operatorname{dim}_{\Phi}$ ad $S=n^{2}-1$. Thus,

$$
n^{2}-1=\operatorname{dim}_{F}\left(\operatorname{Der}_{F} M_{n}(F)\right) \geqslant \operatorname{dim}_{\Phi} \operatorname{Der} S \geqslant \operatorname{dim}_{\Phi} \operatorname{ad}_{S}=n^{2}-1 .
$$

Hence, Der $S=\operatorname{ad}_{S}$ as claimed.

TheOREM 4.12. Assume $n \geqslant 3$ and char $\Phi \neq 2$, 3. Let $\varphi \in$ Aut $K_{n}(A)$ be such that $\varphi\left(e_{i i}-e_{j j}+Z^{\prime}\right)=e_{i i}-e_{j j}+Z^{\prime}$ for all $i, j$. Then there is $a \psi \in(\text { Aut } A)^{\#}$ and an invertible diagonal matrix $u \in M_{n}(N)$ such that $\varphi=\chi_{u} \psi$ where $\chi_{u}$ is conjugation by $u$.

Proof. Just as in the proof of Theorem 4.6, by Lemma 4.5 there exist elements $\alpha_{i j} \in N$ for $i \neq j$ such that $\varphi\left(e_{i j}+Z^{\prime}\right)=\alpha_{i j} e_{i j}+Z^{\prime}$. Only this time applying $\varphi$ to relations (22) and (23) yields

$$
\begin{array}{ll}
\alpha_{i k} \alpha_{k j}=\alpha_{i j} & \text { for } i, k, j \text { distinct, } \\
\alpha_{i j} \alpha_{j i}=1 & \text { for } i \neq j .
\end{array}
$$

Let $u$ be the diagonal matrix with $1, \alpha_{12}, \ldots, \alpha_{1 n}$ down the diagonal. Note that $u^{-1}$ is also diagonal with $1, \alpha_{21}, \ldots, \alpha_{n 1}$ as its diagonal entries. Then $\chi_{u}\left(e_{i j}+Z^{\prime}\right)$ $=u^{-1} e_{i j} u+Z^{\prime}=\alpha_{i 1} \alpha_{1 j} e_{i j}+Z^{\prime}=\alpha_{i j} e_{i j}+Z^{\prime}$. Thus $\psi=\chi_{u}^{-1} \varphi \in$ Aut $K_{n}(A)$ has the property that it fixes each $e_{i j}+Z^{\prime}$ as well as each $e_{i i}-e_{j j}+Z^{\prime}$ for $i \neq j$. 
Beyond this stage the proof is exactly parallel to the proof of Theorem 4.6. One shows the transformations induced from $\psi$ are all equal, and then deduces from (4.5) that the common map, call it $\theta$, belongs to Aut $A$. Finally then, using (4.6) and (4.7) one verifies $\psi=\theta^{\#} \in(\operatorname{Autv} A)^{\#}$.

These conclusions remain valid for any $\varphi \in$ Aut $L_{n}(A)$ (Aut $L_{n}^{\prime}(A)$ ) with the property that $\varphi\left(e_{i i}\right)=e_{i i}\left(\varphi\left(e_{i i}-e_{j j}\right)=e_{i i}-e_{i j}\right)$.

Given any antiautomorphism $\varphi$ of $M_{n}(A),-\varphi$ is an automorphism of $L_{n}(A)$, thus of $L_{n}^{\prime}(A)$ and $K_{n}(A)$ as well. The collection of all such mappings together with Aut $M_{n}(A)$ forms a subgroup of the automorphism group of each of these algebras. It is isomorphic to AAut $M_{n}(A)$, so we will use that notation to denote it.

THEOREM 4.13. If $A$ is finite dimensional over $\Phi$ where char $\Phi \neq 2,3$, and if $n \geqslant 3$, then Aut $M_{n}(A)$ (hence AAut $M_{n}(A)$, also) is of finite index in Aut $L_{n}^{\prime}(A)$ and in Aut $K_{n}(A)$.

Proof. The group $G=$ Aut $L_{n}^{\prime}(A)$ (or Aut $K_{n}(A)$ ) is an algebraic group (see Seligman [11, p. 34]), and $H=$ Aut $M_{n}(A)$ is a closed subgroup of $G$. The Lie algebra of $G$ is Der $K_{n}(A)$ which equals the Lie algebra of $H$, Der $M_{n}(A)$. Therefore, by Proposition 4, p. 33 of Seligman, $H$ is of finite index in $G$.

Now apply Theorem 4.13 and Lemma 4.2 to obtain

Corollary 4.14. With hypotheses as in Theorem 4.13, Aut $M_{n}(A)+$ $\operatorname{LHom}\left(L_{n}(A), Z\right)$ has finite index in Aut $L_{n}(A)$.

REMARK. In the associative case it follows from Martindale [9] that the Lie automorphisms of $R$, a prime ring with a nontrivial idempotent, are precisely AAut $R+\operatorname{LHom}(R, Z)$.

5. $M_{n}(A)$ under the Jordan product. Throughout this section we assume char $\Phi \neq$ 2. Let $J_{n}(A)$ denote the commutative algebra obtained by taking $M_{n}(A)$ under the Jordan product $x \circ y=\frac{1}{2}(x y+y x)$. In this part we investigate the derivation algebra and automorphism group of $J_{n}(A)$. The study of derivations of $J_{n}(A)$ is facilitated by the use of results on transformations in triality. The notion of triality comes up in the study of the Lie algebras of types $D_{4}$ and $F_{4}$ (see Jacobson [5] for example). There the transformations are defined on an octonion algebra, but here we extend the concept to arbitrary algebras.

Let $A$ be an arbitrary algebra with 1 , and assume $\zeta, \eta, \theta$ are linear transformations on $A$ with the property that

$$
\zeta(a b)=\eta(a) b+a \theta(b) \text { for all } a, b \in A .
$$

Then we say $\zeta, \eta, \theta$ are in local triality (or for short, triality). For example if $\zeta=\eta=\theta$, and $\zeta$ is a derivation of $A$, then $\zeta, \eta, \theta$ are in triality.

Lemma 5.1. If $\zeta, \eta, \eta^{\prime}, \theta, \theta^{\prime}$ are transformations on $A$ such that $\zeta, \eta, \theta$ and $\zeta, \eta^{\prime}, \theta^{\prime}$ are in triality then

(1) $\eta^{\prime}=\eta-R_{s}$ where $R_{s}(a)=$ as,

(2) $\theta^{\prime}=\theta+L_{s}$ where $L_{s}(a)=s a$, and $s=\eta(1)-\eta^{\prime}(1)=\theta^{\prime}(1)-\theta(1)$ and $s$ is in the middle nucleus of $A$. 
Proof. Observe that if $\zeta, \eta, \theta$ and $\zeta^{\prime}, \eta^{\prime}, \theta^{\prime}$ are in triality then $\alpha \zeta+\beta \zeta^{\prime}$, $\alpha \eta+\beta \eta^{\prime}, \alpha \theta+\beta \theta^{\prime}$ are in triality for every pair of scalars $\alpha, \beta$. Thus, if $\eta^{\prime \prime}=\eta-$ $\eta^{\prime}, \theta^{\prime \prime}=\theta-\theta^{\prime}$, then $0, \eta^{\prime \prime}, \theta^{\prime \prime}$ are in triality. This says that $\eta^{\prime \prime}(a) b+a \theta^{\prime \prime}(b)=0$ for all $a, b \in A$. Specializing $b$ to equal 1 shows that

$$
\eta^{\prime \prime}(a)=-a \theta^{\prime \prime}(1) \quad \text { for all } a \in A \text {. }
$$

Likewise $a=1$ gives

$$
\theta^{\prime \prime}(b)=-\eta^{\prime \prime}(1) b \quad \text { for all } b \in B \text {. }
$$

If both $a=1$ and $b=1$, then $\eta^{\prime \prime}(1)=-\theta^{\prime \prime}(1)$. Let $s$ be this common value. Then (5.1) and (5.2) shows that $\eta-\eta^{\prime}=\eta^{\prime \prime}=R_{s}$ and $\theta-\theta^{\prime}=\theta^{\prime \prime}=-L_{s}$. Finally, the relation $\eta^{\prime \prime}(a) b+a \theta^{\prime \prime}(b)=0$ implies $(a s) b-a(s b)=0$ as desired.

Lemma 5.2. Let $\partial \in \operatorname{Der}\left(J_{n}(A)\right)$ have the property that $\partial\left(e_{i i}\right)=0$ for $i=1, \ldots, n$. Then $\partial$ induces $n^{2}$ transformations $\partial_{i j}$ on $A$ such that $\partial\left(a e_{i j}\right)=\partial_{i j}(a) e_{i j}$.

Proof. The proof begins just as Lemma 3.5 does. The same argument using the five types of nonzero products and the eigenspaces of the elements $e_{i i}$ demonstrates that $\partial\left(a e_{i i}\right)=\partial_{i i}(a) e_{i i}$ and $\partial\left(a e_{i j}\right)=\partial_{i j}(a) e_{i j}+\partial_{i j}^{\prime}(a) e_{j i}$. In particular, $\partial\left(e_{i j}\right)=\alpha_{i j} e_{i j}+$ $\beta_{j i} e_{j i}$. But since $e_{i j}^{2}=0$, we have $2 e_{i j} \circ \partial\left(e_{i j}\right)=0$. Therefore $\beta_{j i}=0$ for $j \neq i$. Moreover, $e_{i j} \circ a e_{i j}=0$ implies $\alpha_{i j} e_{i j} \circ a e_{i j}+e_{i j} \circ\left(\partial_{i j}(a) e_{i j}+\partial_{i j}^{\prime}(a) e_{j i}\right)=0$, and from this we see $\partial_{i j}^{\prime}(a)=0$ for all $a$. Hence, $\partial\left(a e_{i j}\right)=\partial_{i j}(a) e_{i j}$ in all cases.

Lemma 5.3. Let $\operatorname{char} \Phi \neq 2$, and $n \geqslant 2$. Assume $\partial \in \operatorname{Der} J_{n}(A)$ has the property that $\partial\left(e_{i i}\right)=0$ for $i=1, \ldots, n$. Then there is a diagonal matrix $y \in M_{n}(N)$ such that $\partial-\operatorname{ad}_{y} \in(\operatorname{Der} A)^{\#}$.

Proof. By the preceding lemma $\partial$ induces the transformations $\partial_{i j}$, and for $i, j$ distinct we see from relations (3.2), (3.3), (3.5) that the following transformations are in triality on $A$ :

$$
\begin{array}{lll}
\partial_{i j}, & \partial_{i i}, & \partial_{i j}, \\
\partial_{i j}, & \partial_{i j}, & \partial_{i j}, \\
\partial_{i i}, & \partial_{i j}, & \partial_{j i} .
\end{array}
$$

Let $\alpha_{i j}=\partial_{i j}(1)$. Since relation (3.1) implies $\partial_{i i}$ is a derivation on $A$ under the Jordan product, it follows that $\alpha_{i i}=0$. From (5.5) we conclude that $\alpha_{i j}=-\alpha_{j i}$ for $j \neq i$, so that by Lemma 5.1 we have

$$
\begin{aligned}
& \partial_{i j}=\partial_{i i}+R_{\alpha_{i j}}, \\
& \partial_{j j}=\partial_{i j}-L_{\alpha_{i j}}
\end{aligned}
$$

and also the fact that $\alpha_{i j}$ is in the middle nucleus of $A$. Subtracting (5.4) from (5.3) and then adding (5.5) shows that $\partial_{i i}, \partial_{i i}, \partial_{i j}-\partial_{j j}+\partial_{j i}$ are in triality. However, using (5.6) and (5.7) we may express each of the last three transformations in terms of $\partial_{i i}$. Note that

$$
\partial_{j j}=\partial_{i i}+R_{\alpha_{i j}}-L_{\alpha_{i j}}
$$


and interchanging $j$ and $i$ in (5.7) gives: $\partial_{i i}=\partial_{j i}-L_{\alpha_{j i}}=\partial_{j i}+L_{\alpha_{i j}}$. Therefore, $\partial_{i j}-\partial_{j j}+\partial_{j i}=\partial_{i i}+R_{\alpha_{i j}}-\partial_{i i}-R_{\alpha_{i j}}+L_{\alpha_{i j}}+\partial_{i i}-L_{\alpha_{i j}}=\partial_{i i}$. Hence $\partial_{i i}, \partial_{i i}, \partial_{i i}$ are in triality and $\partial_{i i}$ is a derivation on $A$.

As a consequence of equations (5.3) and (5.6) we have

$$
\partial_{i i}(a b)+(a b) \alpha_{i j}=\partial_{i i}(a) b P \text { ROOF }+a \partial_{i i}(b)+a\left(b \alpha_{i j}\right)
$$

which implies that $\alpha_{i j}$ is in the right nucleus of $A$. Similarly using (5.4) and (5.7) we deduce that $\alpha_{i j}$ is in the left nucleus of $A$. Thus $\alpha_{i j}$ is in the nucleus of $A$. Let $y$ be the diagonal matrix with $0, \alpha_{21}, \ldots, \alpha_{n 1}$ down the diagonal. From equations (5.6), (5.7), (5.8) it follows that $\partial_{1 j}(a)=\partial_{11}(a)-a \alpha_{j 1}, \partial_{i 1}(a)=\partial_{11}(a)+\alpha_{i 1} a$ and $\partial_{i j}(a)=$ $\partial_{11}(a)+\left[\alpha_{j 1}, a\right]$. If $n \geqslant 3$, then for $i, j$ distinct from $1, \partial_{i j}, \partial_{i 1}, \partial_{1 j}$ are in triality and $\partial_{i j}(a)=\partial_{11}(a)+\alpha_{i 1} a-a \alpha_{j 1}$. We conclude from these relations that $\partial=\left(\partial_{11}\right)^{\#}+$ $\operatorname{ad}_{y}$ where $\partial_{11} \in \operatorname{Der} A$.

LemmA 5.4. Let $\operatorname{char} \Phi \neq 2$, and $n \geqslant 2$. Suppose $\partial \in \operatorname{Der} J_{n}(A)$. Then there is a $w \in M_{n}(N)$ such that $\partial^{\prime}=\partial-\operatorname{ad}_{w}$ has the property that $\partial^{\prime}\left(e_{i i}\right)=0$ for all $i$.

Proof. Apply $\partial$ to the relation $e_{i i} \circ e_{i i}=e_{i i}$ to conclude that $\partial\left(e_{i i}\right)$ lies in the $\frac{1}{2}$-eigenspace relative to multiplication by $e_{i i}$. Therefore we may write

$$
\partial\left(e_{i i}\right)=\sum_{k \neq i} \beta_{k i}^{i} e_{k i}+\sum_{l \neq i} \beta_{i l}^{i} e_{i l}
$$

Now if $j \neq i$, then $\partial\left(e_{i i}\right) \circ e_{i j}+e_{i i} \circ \partial\left(e_{i j}\right)=0$, and from this we calculate that

$$
\beta_{j i}^{i}=-\beta_{j i}^{j} \text {. }
$$

Thus, if $\gamma_{k l}=\beta_{k l}^{l}$ for $k \neq l$ and if $\gamma_{k k}=0$, then $w=\sum \gamma_{k l} e_{k l}$ has the property that

$$
\begin{aligned}
\operatorname{ad}_{w}\left(e_{i i}\right) & =\sum_{k} \gamma_{k i} e_{k i}-\sum_{l} \gamma_{i l} e_{i l}=\sum_{k \neq i} \beta_{k i}^{i} e_{k i}-\sum_{l \neq i} \beta_{i l}^{l} e_{i l} \\
& =\sum_{k \neq i} \beta_{k i}^{i} e_{k i}+\sum_{l \neq i} \beta_{i l}^{i} e_{i l}=\partial\left(e_{i i}\right) .
\end{aligned}
$$

The proof will be complete once it is proven that each $\gamma_{k l} \in N$, and hence $\operatorname{ad}_{w} \in \operatorname{Der} J_{n}(A)$. A computation of $\partial$ applied to $a e_{i i} \circ e_{i i}=a e_{i i}$ shows that

$$
\partial\left(a e_{i i}\right)=\sum_{k \neq i} \beta_{k i}^{i} a e_{k i}+\sum_{l \neq i} a \beta_{i l}^{i} e_{i l}+\Delta(a, i) e_{i i}
$$

Using this relation, we conclude from $\partial$ acting on $a e_{i i} \circ b e_{j j}=0$ that

$$
0=\frac{1}{2}\left\{\left(a \beta_{i j}^{i}\right) b+a\left(\beta_{i j}^{j} b\right)\right\} e_{i j}+\frac{1}{2}\left\{b\left(\beta_{j i}^{i} a\right)+\left(b \beta_{j i}^{j}\right) a\right\} e_{j i} \text {. }
$$

Since $\beta_{i j}^{i}=-\beta_{j i}^{j}$, this implies $\gamma_{i j}=\beta_{i j}^{j}$ is in the middle nucleus of $A$.

Let us apply $\partial$ to both sides of $e_{i i} \circ b e_{i j}=\frac{1}{2} b e_{i j}$. Now

$$
\partial\left(e_{i i}\right) \circ b e_{i j}=\frac{1}{2} \sum_{k \neq i} \beta_{k i}^{i} b e_{k j}+b \beta_{j i}^{i} e_{i i}
$$

and $e_{i i} \circ \partial\left(b e_{i j}\right)$ has nonzero entries just in the $i$ th row and column. Thus,

$$
\partial\left(b e_{i j}\right)=\sum_{k \neq i} \beta_{k i}^{i} b e_{k j}+\sum_{l \neq i} c_{i l} e_{i l}+\sum_{l} c_{l i} e_{l i} .
$$


The next step is to equate coefficients after applying $\partial$ to $e_{i j} \circ b e_{i j}=\frac{1}{2} b e_{i j}$. The result obtained is

$$
\partial\left(b e_{i j}\right)=\sum_{k \neq i} \beta_{k i}^{i} b e_{k j}+\sum_{l \neq j} b \beta_{j l}^{j} e_{i l}+\Delta(b, i, j) e_{i j}+\Delta^{\prime}(b, j, i) e_{j i}
$$

Then $\partial\left(a e_{i i}\right) \circ b e_{i j}+a e_{i i} \circ \partial\left(b e_{i j}\right)=\frac{1}{2} \partial\left(a b e_{i j}\right)$ implies $\left(\beta_{k i}^{i} a\right) b=\beta_{k i}^{i}(a b)$ and $a\left(b \beta_{j i}^{j}\right)$ $=(a b) \beta_{j l}^{j}$. Thus, the $\gamma$ 's lie in $N, w=\sum \gamma_{k l} e_{k l}$ in $M_{n}(N)$, and $\operatorname{ad}_{w}$ in $\operatorname{Der} J_{n}(A)$. As noted above, then for $\partial^{\prime}=\partial-\operatorname{ad}_{w}$, we have $\partial^{\prime}\left(e_{i i}\right)=0$ for all $i$.

The net effect of Lemmas 5.3 and 5.4 is a proof of

THEOREM 5.5. Let char $\Phi \neq 2$. Then

$$
\operatorname{Der} J_{n}(A)=(\operatorname{Der} A)^{\#}+\operatorname{ad}_{M_{n}(N)}=\operatorname{Der} M_{n}(A) \text {. }
$$

REMARKs. Recall that the last equality follows from Corollary 4.9 and the comments preceding Corollary 4.11.

Previous results analogous to Theorem 5.5 have been known to hold in the associative case. Jacobson and Rickart showed in [6, Theorems 7 and 22] that $\operatorname{Der} M_{n}(A)=\operatorname{Der} J_{n}(A)$ when $A$ is associative, while Herstein [2, p. 55] proved that every Jordan derivation of a prime associative ring $R$ is a derivation of $R$, except when $R$ is a commutative integral domain of characteristic 2 . These results would have been useful had we known ab initio that $\partial \in \operatorname{Der} J_{n}(A)$ implies $\partial\left(M_{n}(N)\right) \subseteq$ $M_{n}(N)$. Indeed the major effort of Lemma 5.4 is in proving the image of each $e_{i i}$ lies in $M_{n}(N)$.

The work on the next theorem concerning Aut $J_{n}(A)$ has largely been done already, but we collect the results for the convenience of the reader.

Theorem 5.6. Assume char $\Phi \neq 2$. Let $G=\left\{\psi \in\right.$ Aut $J_{n}(A) \mid \psi\left(e_{i i}\right)=e_{i i}$ for all $\left.i\right\}$ and $H=\left\{\psi \in\right.$ Aut $\left.J_{n}(A) \mid \psi\left(J_{n}(N)\right) \subseteq J_{n}(N)\right\}$. Then

$$
\text { G }
$$

$$
\text { Aut } M_{n}(A) \subseteq \operatorname{GL}(n, N) \cdot(\text { GAut } A)^{\#} \subseteq \text { GAut } M_{n}(A) \subseteq H \subseteq \text { Aut } J_{n}(A) .
$$

(ii) $G=X \cdot(\text { GAut } A)^{\#}$ where $X$ is the subgroup consisting of conjugations by diagonal matrices with entries in $N$.

(iii) If $N$ is Artinian, then $\mathrm{GL}(n, N) \cdot(\mathrm{GAut} A)^{\#}=$ GAut $M_{n}(A)=H$.

(iv) If $A$ is finite dimensional, then Aut $M_{n}(A)$, hence GAut $M_{n}(A)$ also, is of finite index in Aut $J_{n}(A)$.

Proof. Assertions (i) and (ii) are consequences of results in \$3, notably Theorem 3.6 and Lemma 3.12. Part (iii) follows from Lemma 3.4 and Theorems 3.6 and 3.13. Since $\operatorname{Der} J_{n}(A)=\operatorname{Der} M_{n}(A)$, statement (iv) can be concluded by the same algebraic group argument used in $\$ 4$.

REMARKS. For associative rings Herstein $[2$, p. 50] has shown that if $\varphi$ is a Jordan homomorphism of $R$ onto a prime ring $R^{\prime}$, then $\varphi$ is a homomorphism or an antihomomorphism. This result is false in the nonassociative case as the next example illustrates. 
Choose $\lambda, \mu \in \Phi$ such that $\lambda+\mu=1$, and let $B$ have basis $\left\{\varepsilon_{i j}\right\}$ for $i, j=$ $1, \ldots, n$ where the product in $B$ is given by $\varepsilon_{i j} * \varepsilon_{k l}=\delta_{j k} \lambda \varepsilon_{i l}+\delta_{i l} \mu \varepsilon_{k j}$. If $B_{J}$ denotes $B$ under the Jordan product then $B_{J}$ is Jordan isomorphic to $M_{n}(\Phi)$ under the correspondence $\varepsilon_{i j} \rightarrow e_{i j}$. Since $B$ is nonassociative when $\lambda \neq 1,0$, this map is neither an isomorphism nor an anti-isomorphism of $B$ onto $M_{n}(\Phi)$.

Now if $A$ is any algebra then $M_{n}(A) \approx A \otimes M_{n}(\Phi)$. If in addition $A$ is assumed to be commutative then $J_{n}(A) \approx A \otimes J_{n}(\Phi)$. If $A$ is any commutative algebra isomorphic to $J_{n}(\Phi)$, such as $B_{J}$ above, then there is an automorphism of $J_{n}(A)$ mapping $A \otimes 1$ to $1 \otimes J_{n}(\Phi)$. If the nucleus of $A$ is $\Phi 1$, as with $B_{J}$, then such an automorphism is not in Aut $M_{n}(A)$ or GAut $M_{n}(A)$ since it fails to preserve the nucleus which is $1 \otimes M_{n}(\Phi)$. By taking $A$ to be the tensor product of any number of algebras isomorphic to $J_{n}(\Phi)$, we see that the index of Aut $M_{n}(A)$ in Aut $J_{n}(A)$ can be arbitrarily large.

We conclude our remarks with one additional example. Suppose $B$ is the algebra above, and form the algebra $J_{n}(B)$. The product is given by

$$
2 \varepsilon_{i j} e_{q r}^{\circ} \varepsilon_{k l} e_{s t}=\delta_{r s} \delta_{j k} \lambda \varepsilon_{i l} e_{q t}+\delta_{r s} \delta_{i l} \mu \varepsilon_{k j} e_{q t}+\delta_{t q} \delta_{j k} \mu \varepsilon_{i l} e_{s r}+\delta_{t q} \delta_{i l} \lambda \varepsilon_{k j} e_{s r}
$$

Using this formula one can show that the map $\varphi$ given by $\varphi\left(\varepsilon_{i j} e_{q r}\right)=\varepsilon_{q r} e_{i j}$ belongs to Aut $J_{n}(B)$. If $\lambda \neq 1,0$ then $\varphi$ interchanges the nonassociative algebra $B$ with $M_{n}(\Phi)$; hence it fails to belong to Aut $M_{n}(B)$. This example illustrates that the coefficient algebra $B$ need not be commutative to make interchanges of this sort possible.

\section{REFERENCES}

1. G. Domokos and S. Kovesi-Domokos, The algebra of color J. Math. Phys. 14 (1973), 1651-1667.

2. I. N. Herstein, Topics in ring theory, Univ. of Chicago Press, Chicago, Ill., 1965.

3. N. Jacobson, Abstract derivations and Lie algebras, Trans. Amer. Math. Soc. 42 (1937), 206-224.

4. ___ Structure of alternative and Jordan bimodules, Osaka J. Math. 6 (1954), 1-71.

5. —_ Exceptional Lie algebras, Marcel Dekker, New York, 1971.

6. N. Jacobson and C. E. Rickart, Jordan homomorphisms of rings, Trans. Amer. Math. Soc. 69 (1950), 479-502.

7. L. S. Levy, Artinian, non-Noetherian rings, J. Algebra 47 (1977), 276-304.

8. W. S. Martindale III, Lie derivations of primitive rings, Michigan Math. J. 11 (1964), 183-187.

9. __ Lie isomorphisms of prime rings, Trans. Amer. Math. Soc. 142 (1969), 437-455.

10. A. Rosenberg and D. Zelinsky, Automorphisms of separable algebras, Pacific J. Math. 11 (1961), 1109-1117.

11. G. B. Seligman, Algebraic groups, Yale University, mimeographed notes, 1964.

Department of Mathematics, University of Wisconsin, Madison, Wisconsin 53706 